\title{
Características del talento matemático asociadas a la invención de problemas ${ }^{1}$
}

\author{
Features associated mathematical talents to the invention of problems
}

\section{Caracteriza talentos matemáticos associados com a invenção de problemas}

Recibido: mayo de 2013

Aprobado: agosto de 2013
Johan Espinoza González ${ }^{2}$

Jose Luis Lupiañez Gómez ${ }^{3}$

Isidoro Segovia Alex ${ }^{4}$

\section{Resumen}

En este documento se analizan las características del talento matemático en una muestra de 21 estudiantes considerados con talento matemático que participan en el proyecto ESTALMAT Andalucía. El estudio se centra especialmente en la invención de problemas aritméticos el cual permite a los estudiantes poner en prácticas sus conocimientos, habilidades, creatividad, originalidad, capacidad de generalización, entre otros. Se encontró que los estudiantes con talento matemático elaboran problemas extensos, con variedad de cantidades y tipos de números, con proposiciones interrogativas de asignación, de estructura mixta y con tres o más relaciones semánticas distintas. De igual forma, dichos problemas requieren cuatro o más pasos para ser resueltos y presentan dos o más procesos de cálculo distintos.

Palabras clave: Otras nociones de Educación Matemática; resolución de problemas; planteamiento de problemas; alumno; necesidades especiales; alumnos con talento matemático; problemas aritméticos.

\begin{abstract}
This paper analyzes the characteristics of mathematical talent in a sample of 21 students considered with mathematical talent involved in the project ESTALMAT Andalusia. The study focuses particularly on the invention of arithmetic problems which allows students to put into practice their knowledge, skills, creativity, originality, generalizability, among others. They found that students with mathematical talent drawn widespread problems with a variety of amounts and types of numbers, with interrogative propositions allocation of mixed structure with three or more different semantic relations. Similarly, these problems require four or more steps to be resolved and have two or more different calculation processes.
\end{abstract}

1 Artículo de Investigación.

2 Universidad Nacional, Costa Ricao. Contacto: jespinoza@una.cr

3 Universidad de Granada, España. Contacto: lupi@ugr.es.

4 Universidad de Granada, España. Contacto: isegovia@ugr.es. 
Keywords: Other notions of mathematics education, problem solving, problem posing, student, special needs students with mathematical talent; arithmetic problems.

\section{Resumo}

Este artigo analisa as características de talento matemático em uma amostra de 21 alunos considerados com talento matemático envolvido no projeto ESTALMAT Andaluzia. O estudo centra-se especialmente sobre a invenção de problemas aritméticos que permite aos alunos colocar em prática os seus conhecimentos, habilidades, criatividade, originalidade, generalização, entre outros. Eles descobriram que os alunos com talento matemático elaborado problemas generalizados com uma variedade de quantidades e tipos de números, com interrogativa proposições alocação de estrutura mista, com três ou mais relações semânticas diferentes. Da mesma forma, esses problemas exigem quatro ou mais passos a serem resolvidos e têm dois ou mais processos de cálculo diferentes.

Palavras-chave: Outras noções de educação matemática, resolução de problemas, problema levantamento, estudantes, alunos com necessidades especiais, com talento matemático, problemas de aritmética.

\section{Presentación del problema}

Esta investigación comprende dos campos de estudio. Uno de ellos es la invención de problemas matemáticos, la cual surge como línea de investigación a partir de estudios realizados sobre la Resolución de Problemas (Castro, 2008) y es tal que algunos distinguidos investigadores e instituciones en Educación Matemática (Ellerton, 1986; Krutetskii, 1976; Freudenthal, 1973; Brown \& Walter, 1990; NCTM 1998, 2000), la reconocen como actividad importante de la experiencia matemática de cualquier estudiante.

El término invención de problemas ha sido empleado generalmente para referirse tanto a la generación de nuevos problemas como a la reformulación de problemas dados (Silver, 1994; English, 1997; Silver \& Cai, 1996). Uno de los primeros autores en referirse a este proceso fue Dunker (citado por Silver, 1994), quien menciona que la resolución de problemas consiste en sucesivas reformulaciones de un problema inicial, el cual ocurre durante el proceso de resolución de un problema complejo (Silver, Mamona-Down, Leung \& Kenny, 1996). Sin embargo, la invención de problemas también puede ocurrir antes o después del proceso de solución de un problema (Silver, 1994).

El otro campo de estudio corresponde al talento matemático, cuya investigación es reciente y se ha centrado en tres grandes focos: la caracterización del talento matemático, el establecer mecanismos de identificación y ofrecer alternativas de intervención (Castro, 2008). Centrándonos en la caracterización del talento matemático, encontramos que algunos investigadores (Krutetskii, 1976; Greenes, 1981; House, 1987; Johnson, 1983; Sriraman, 2003; Pasarín, Feijoo, Díaz \& Rodríguez, 2004; Lee, 2005) se han preocupado por observar y analizar el pensamiento característico de este tipo de estudiantes en diferentes tareas de resolución de problemas, resultando que algunas de ellas están relacionadas con la invención de problemas. Sin embargo, son pocas los estudios que abordan los procesos de invención de problemas con estudiantes considerados con talento matemático y específicamente el estudio de las características del talento matemático asociadas a dichos procesos. A pesar de esto, consideramos que hay elementos teóricos que sustentan el estudio del talento matemático 
mediante la invención de problemas, ya que este tipo de actividades proporciona una herramienta poderosa para estudiar niños con talento matemático (Ellerton, 1986) y sus habilidades matemáticas (Kesan et al., 2010), así como una características de la actividad creativa o talento excepcional y una "ventana" que permite observar la comprensión matemática de los estudiantes (Silver, 1994).

Así, nuestro problema de investigación consiste en determinar las características de los problemas planteados por un grupo de estudiantes con talento matemático ante dos tareas de invención de problemas aritméticos verbales. Para ellos, nos propusimos construir un instrumento de planteamiento de problemas con dos tareas o situaciones semiestructuradas de invención problemas aritméticos verbales; desarrollar y utilizar un esquema analítico para valorar los problemas aritméticos planteados por los estudiantes, definir categorías de análisis que permitan caracterizar las producciones de los estudiantes y caracterizar los problemas planteados por los estudiantes de la muestra con base en las categorías de análisis definidas.

\section{Metodología}

Esta investigación es de tipo exploratorio descriptivo pues corresponde a un primer acercamiento al estudio de la invención de problemas aritméticos por estudiantes considerados con talento matemático, privilegiando la descripción e interpretación de la información pero al mismo tiempo dando un tratamiento cuantitativo a los datos (Espinoza,

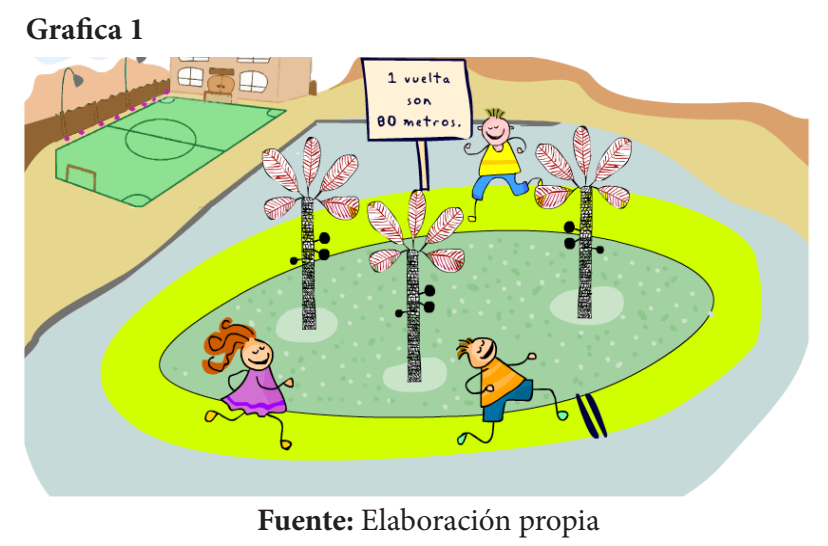

5 http://ntic.educacion.es/w3/eos/MaterialesEducativos/mem2009/problematic/menuppal.html
2011). Los sujetos de estudio corresponden a un grupo de 21 estudiantes considerados con talento matemático que participaron en el proyecto ESTALMAT Andalucía durante el curso 20102011 y que tienen edades comprendidas entre los 13 y los 15 .

Descripción del instrumento para recolectar información. En este estudio se elaboró un cuestionario con dos tareas o situaciones semiestructuradas de invención de problemas (Stoyanova, 1998) con características diferentes. La primera tarea plantea lo siguiente: De acuerdo con la información de la siguiente figura, inventa un problema matemático que te parezca difícil de resolver y que en su resolución se utilice una o varias de las operaciones de suma, resta, multiplicación o división. Si lo consideras necesario puedes agregar más datos o información. La figura propuesta ${ }^{5}$ a los estudiantes para que inventen el problema.

Las indicaciones de la segunda tarea de invención de problemas son similares a la anterior; pero en ésta tuvieron que inventar un problema matemático a partir de la siguiente situación expuesta de forma textual: Un tren con cuatro vagones para pasajeros sale de una estación a las 9:00 h con destino a Málaga. El tren tiene una capacidad máxima para 294 pasajeros.

Descripción de las categorías de análisis empleadas. Para elaborar las categorías de análisis consideramos las características propias de esta investigación y realizamos una revisión de las variables de estudio de los problemas aritméticos (Puig \& Cerdán, 1988; Web, 1997; Castro, 1995; Silver \& Cai, 2005; Rico et al., 1988) y los esquemas empleados en otras investigaciones para valorar las producciones de los estudiantes ante tareas de invención de problemas (Leung \& Silver, 1997; Silver \& Cai, 2005; Cázares, 2000; Ayllón, 2004; Silver \& Cai, 1996). Así, definimos tres categorías de análisis y en cada una de ellas variables de estudio. Dichas variables se explican con mayor detalle y sustento teórico en el trabajo de investigación de Espinoza (2011). 
En la primera categoría, denominada estructura sintáctica, se estudió la longitud del enunciado, tipo de proposición interrogativa y tipo de número empleado. La segunda categoría llamada estructura matemática fue analizada de acuerdo con el tipo de estructura operatoria y número de etapas, cantidad de procesos de cálculo distintos implicados en la solución del problema y cantidad de pasos distintos para resolver el problema. Por último, en la categoría de estructura semántica, los problemas fueron estudiados en relación a su estructura semántica y cantidad de relaciones semánticas distintas presentes en el enunciado.

Esquema para valorar las producciones de los estudiantes

Todas las producciones de los estudiantes correspondieron a problemas matemáticos, por lo que los autores del estudio los resolvieron y clasificaron en resolubles y no resolubles. Dentro de las producciones de los estudiantes encontramos problemas matemáticos no resolubles que presentaron características importantes de analizar. Por ello los clasificamos como incompletos (Puig \& Cerdán, 1988) y los distinguimos de aquellos que presentan incompatibilidad matemática de tipo numérico o conceptual (Espinoza, 2011). A los problemas matemáticos resolubles y no resolubles clasificados como incompletos o que presentan incompatibilidad matemática de tipo numérica se les aplicó el análisis de la estructura sintáctica, semántica y matemática explicado anteriormente. Mientras que los problemas matemáticos que presentan incompatibilidad matemática de tipo conceptual fueron analizados sólo desde su estructura sintáctica, pues no era posible analizar la estructura semántica y matemática.

\section{Resultados}

En primera instancia encontramos que todos los estudiantes respondieron a las dos tareas de invención de problemas propuestas, obteniendo un total de 42 problemas matemáticos de los cuales el 57\% son resolubles. Con respecto a los problemas no resolubles, resultó que el $66,6 \%$ presentan incompatibilidad matemática y el $33,3 \%$ son incompletos.
Con respecto al análisis según la estructura sintáctica, resultó que el promedio de la cantidad de proposiciones presentes en los problemas fue de 5,27 y que el $69,1 \%$ de los problemas están conformados por cinco o más proposiciones. También se observó que la mayoría de las proposiciones interrogativas son de asignación (52,4\%), mientras que las proposiciones interrogativas relacionales fueron las menos preferidas. Por último, los enunciados se caracterizaron por la presencia de números naturales $(97,6 \%)$ y números racionales expresados tanto en notación decimal como fraccionaria $(43,9 \%)$

En relación con el análisis según la estructura matemática, se encontró que los estudiantes prefirieron plantear problemas de estructura mixta (80\%), seguido de problemas de estructura multiplicativa (17,5\%). Estos dos resultados nos indican que el 97,5\% de problemas son de estructura multiplicativa o aditiva de más de una etapa o problemas de estructura mixta. También observamos que el 92,5\% de los problemas implicaron dos o más procesos distintos para ser resueltos y que aproximadamente la mitad $(47,5 \%)$ de los problemas presentan tres o más procesos distintos. Además se observó que los problemas requieren en promedio 3,95 pasos para ser resueltos. Esto se evidenció en la cantidad de problemas que requieren cuatro o más pasos para ser resueltos, puesto que el 67,5\% presentaron esta característica. Otro resultado a destacar es que el $66,6 \%$ de los problemas que presentan dos o tres relaciones semánticas distintas implican cuatro o más pasos para ser resuelto y que el $60,7 \%$ de los problemas que incluyen dos o tres procesos requieren cuatro o más pasos para ser resueltos.

Por último, en el análisis según la estructura semántica, resultó que los estudiantes sólo plantearon un problema aditivo y presenta la relación semántica de cambio. Con respecto a los 33 problemas de estructura aditiva y mixta, se encontró que el $81,8 \%$ son de combinación, el 51,5\% de cambio y en menor proporción $(9,1 \%)$ presentaron la estructura semántica de igualación. De los 7 problemas de estructura multiplicativa, 5 son de producto de medida, 5 de isomorfismo de medida y sólo uno es de comparación multiplicativa. Con respecto 
a los 39 problemas de estructura multiplicativa y mixta, resultó que el 79,5\% son de isomorfismo de medida y el $33,3 \%$ de producto de medidas. Otro resultado destacable es que los problemas presentan en promedio 2,83 relaciones semánticas distintas y que el $65 \%$ presentan tres o más relaciones semánticas distintas.

\section{Conclusiones}

Luego de revisar los problemas aritméticos planteados por los estudiantes en relación con las variables de estudio definidas y considerando las limitaciones de nuestra investigación, podemos concluir que existen algunas características del talento matemático que se asocian a los procesos de invención de problemas aritméticos. Entre ellas encontramos que los estudiantes con talento matemático de la muestra se caracterizaron por inventar una gran cantidad de problemas no resolubles; incluir en el enunciado del problema cinco o más proposiciones; emplear números naturales y en menor proporción números racionales; utilizar dos tipos de números distintos, ya sean naturales o racionales expresados en notación decimal y/o fraccionaria; incluir como pregunta del problema proposiciones interrogativas de asignación; plantear problemas de estructura mixta; incluir las relaciones semánticas de combinación y producto de medidas; plantear tres o más relaciones semánticas distintas; inventar problemas que requieren cuatro o más pasos para ser resueltos y que presentan dos o más procesos de cálculo distintos en su solución.

\section{Referencias}

Castro, E (2008). Resolución de problemas: ideas, tendencias e influencias en España. En R. Luengo, B. Gómez, M. Camacho \& B. Lorenzo (Eds.), Investigación en Educación Matemática XII (pp 113-140). Badajoz: Sociedad Española de Investigación en Educación Matemática, SEIEM.
Cázares, J. (2000). La invención de problemas en escolares de primaria: un estudio evolutivo. Memoria de tercer ciclo. Granada: Universidad de Granada

Ellerton, N. F. (1986). Children's made up mathematics problems: A new perspective on talented mathematicians. Educational Studies in Mathematics, 17, 261-271.

English, L. D. (1997). The development of fifth grade children's problem posing abilities. Educational Studies in Mathematics, 34, 183-217.

Espinoza, J. (2011). Invención de problemas aritméticos por estudiantes con talento matemático: Un estudio exploratorio. Memoria de Tercer Ciclo. Granada: Universidad de Granada

Greenes, C. (1981). Identifying the gifted student in mathematic. Arithmetic Teacher, 28 (8), 14-17.

Kesan, C., Kaya, D \& Güvercin, S. (2010). The effect of problem posing approach to the gifted student's mathematical abilities. International Online Journal of Educational Sciences, 2(3), 677-687.

Krutetskii, V.A (1976). The psychology of mathematical abilities in school children. Chicago: University of Chicago Press.

Leung, S. K., \& Silver, E. A. (1997). The role of task format, mathematics knowledge, and creative thinking on the arithmetic problem posing of prospective elementary school teachers. Mathematics Education Research Journal, 9(1), 5-24.

Polya, G. (1954). Mathematics and plausible reasoning. Princenton, NJ: Princenton University Press. 
Puig, L., Cerdán, F. (1988). Problemas aritméticos. Madrid: Síntesis.

Silver, E. A. (1994). On mathematical problem posing. For the Learning of Mathematics, 14(1), 19-28.

Silver, E., \& Cai, J. (1996). An analysis of arithmetic problem posing by middle school students. Journal for Research in Mathematics Education, 27(5), 521-539.
Silver, E., \& Cai, J. (2005). Assessing students' mathematical problem posing. Teaching Children Mathematics, 12(3), 129-135.

Stoyanova, E. (1998). Problem posing in mathematics classrooms. En A. McIntosh \& N. Ellerton (Eds.), Research in Mathematics Education: a contemporary perspective (pp 164-185). MASTEC: Edit Cowan University. 